\title{
The pathology and treatment of cardiac arrhythmias: focus on atrial fibrillation
}

\author{
This article was published in the following Dove Press journal: \\ Vascular Health and Risk Management \\ 30 March 20II \\ Number of times this article has been viewed
}

\section{Constanze Schmidt* \\ Jana Kisselbach* \\ Patrick A Schweizer \\ Hugo A Katus \\ Dierk Thomas}

Department of Cardiology, Medical University Hospital, Heidelberg, Germany

"These authors contributed equally to this work
Correspondence: Dierk Thomas

Department of Cardiology,

Medical University Hospital,

Heidelberg, Im Neuenheimer

Feld 410, D-69120 Heidelberg,

Germany

Tel +49 622I 568855

Fax +49 622I 5655I4

Email dierk.thomas@med.uni-heidelberg.de
Abstract: Atrial fibrillation (AF) is the most frequently encountered sustained cardiac arrhythmia in clinical practice and a major cause of morbidity and mortality. Effective treatment of AF still remains an unmet medical need. Treatment of AF is based on drug therapy and ablative strategies. Antiarrhythmic drug therapy is limited by a relatively high recurrence rate and proarrhythmic side effects. Catheter ablation suppresses paroxysmal AF in the majority of patients without structural heart disease but is more difficult to achieve in patients with persistent AF or with concomitant cardiac disease. Stroke is a potentially devastating complication of $\mathrm{AF}$, requiring anticoagulation that harbors the risk of bleeding. In search of novel treatment modalities, targeted pharmacological treatment and gene therapy offer the potential for greater selectivity than conventional small-molecule or interventional approaches. This paper summarizes the current understanding of molecular mechanisms underlying AF. Established drug therapy and interventional treatment of AF is reviewed, and emerging clinical and experimental therapeutic approaches are highlighted.

Keywords: atrial fibrillation, antiarrhythmic therapy, anticoagulation, catheter ablation, stroke

\section{Introduction}

Atrial fibrillation (AF) is the most common sustained cardiac arrhythmia. The arrhythmia has a prevalence of $1 \%$ and is age-dependent with $\sim 10 \%$ of patients $>80$ years being affected in contrast to $0.1 \%$ of all individuals $<55$ years. ${ }^{1-3}$ The increasing epidemiological significance of $\mathrm{AF}$ is further illustrated by a projected calculation predicting that AF prevalence may have increased $\geq 2.5$-fold by the year $2050 .{ }^{2}$ In elderly patients the development of AF is mostly related to cardiac disorders, whereas younger patients may develop AF in the absence of underlying heart disease ("lone AF"). In addition, gender-specific pathophysiological mechanisms are suggested by retrospective data. Between 1970 and 1990 the prevalence in men has more than doubled, while the prevalence of AF in women during this period remained unchanged. Furthermore, the annual incidence of $\mathrm{AF}$ is between $0.9 \%$ and $1.8 \%$ for men aged 65 to 74 and $1.8 \%$ and $4.3 \%$ for men aged 75 to 84 . Corresponding incidences for women are lower and vary between $0.5 \%$ and $2.2 \%$ per 100 person-years, respectively. ${ }^{4} \mathrm{AF}$ is associated with a 2-fold age-independent increase in mortality which is directly attributed to the arrhythmia as opposed to concomitant cardiovascular conditions. ${ }^{5}$

Symptoms associated with AF are primarily caused by rapid and irregular heartbeat and include palpitations, dizziness, lightheadedness, anxiety, and reduced exercise capacity which result in severely impaired quality of life. ${ }^{6}$ However, one-third of 
patients exhibit no symptoms and are unaware of abnormal heart rhythm, preventing early detection and timely introduction of therapies. In a subgroup of patients the severity of symptoms decreases with time owing to a transition from paroxysmal to permanent $\mathrm{AF}^{2}{ }^{2}$ In predisposed individuals the arrhythmia may be triggered by cardiac or thoracic surgery, infections, alcohol intake (binge drinking; "holiday heart disease"), nicotine or caffeine, hypoglycemia, electrolyte imbalances, and physical or emotional stress.

Recent in vitro and in vivo evidence provided significant advances towards a comprehensive understanding of structural and electrical mechanisms underlying AF on the molecular level. ${ }^{7,8}$ Based on these data, interventional and pharmacological therapies targeting novel mechanisms and utilizing innovative modalities (eg, gene or stem cell therapy) are currently being developed and evaluated in pre-clinical and clinical studies. ${ }^{9,10}$ In the present review basic mechanistic knowledge is briefly summarized. We then focus on current and newly emerging pharmacological and interventional approaches to pharmacological antiarrhythmic treatment, catheter ablation, stroke prevention, and so-called "upstream therapy" (ie, reversal of atrial remodeling by targeting substrate development).

\section{Pathology}

In recent years the pathophysiology of AF has been studied extensively. Two basic electrophysiological mechanisms are proposed to underlie initiation and perpetuation of AF (Figure 1). First, rapid ectopic activity may trigger and maintain $\mathrm{AF}^{7}$ Second, sustained AF may depend on

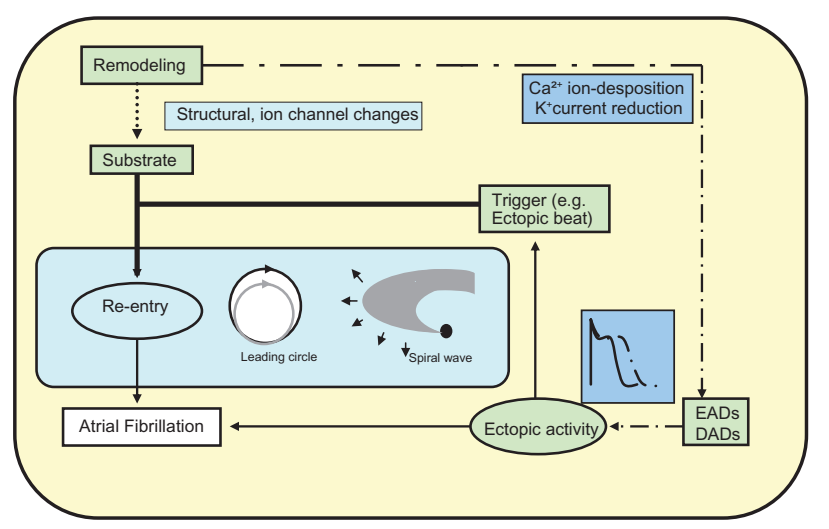

Figure I Electrical mechanisms of atrial fibrillation (AF). Rapid ectopic activity and re-entry are key electrophysiological mechanisms of AF. The development of functional re-entrant circuits requires a trigger (eg, an ectopic beat) that initiates the arrhythmia. Atrial remodeling creates a structural substrate for re-entry by altering ion channel function or by inducing fibrosis. The leading circle concept and the spiral wave hypothesis represent models of re-entry. Remodeling may induce ectopic electrical discharges through changes in $\mathrm{Ca}^{2+}$ ion desposition or $\mathrm{K}^{+}$current levels, resulting in trigger activity.

Abbreviations: EAD, early after depolarization; DAD, delayed after depolarization. single or multiple electrical re-entrant circuits resulting from shortening of effective refractory periods and from localized deceleration of intra-atrial conduction. In addition to initiation by electrical trigger beats, re-entry requires a susceptible substrate such as fibrosis. Both mechanisms are not mutually exclusive. Rather, trigger activity and re-entry co-exist and influence each other, providing a mechanistic basis for the progressive nature of the disease. In addition to electroanatomical mechanisms, genetic mutations as well as several loci associated with AF have been identified through analysis of rare monogenetic hereditary AF or using genomewide association studies, respectively. ${ }^{11-17}$ Affected genes or regulatory elements and their pathophysiological roles are not yet identified in the majority of cases.

\section{Focal electrical activity}

Ectopic impulse formation is caused by localized discharges from electrical "pacemakers" arising from an area other than the sinus node. In AF, pulmonary veins exhibit electrical activity that triggers the arrhythmia. ${ }^{18}$ Automaticity may be attributed to an imbalance of cellular repolarizing and depolarizing ionic currents in the diastolic phase of the action potential which depends on the adrenergic tone. If diastolic depolarization is increased, the cell reaches the threshold for premature action potential firing, and generates ectopic activity. In addition, early or delayed after depolarizations (EAD or DAD) may depolarize a cell before the next expected normal action potential and cause premature action potential firing. Early after depolarization results from a prolongation of the action potential duration owing to reduced repolarizing $\mathrm{K}^{+}$currents. Delayed after depolarizations are caused by spontaneous diastolic release of $\mathrm{Ca}^{2+}$ ions from the sarcoplasmic reticulum (SR), which may be due to SR calcium overload or to dysfunctional $\mathrm{Ca}^{2+}$ release through channels in the sarcoplasmic reticulum.

\section{Mechanisms of re-entry}

At the molecular level, re-entry may arise from two different pathophysiological mechanisms (Figure 1). First, re-entry and the development of AF may depend on the local balance between cellular refractoriness and conduction velocity ("leading circle idea"). ${ }^{7,8} 19$ If refractoriness is short and the intercellular transmission of action potentials is slow, the likelihood of circulating impulses after a distinct single re-entry circuit is increased. The re-entrant circuit terminates when the effective refractory period is prolonged or when impulse propagation is accelerated, forcing the excitation wave front into tissue that is still refractory. According to a 
second model ("spiral wave theory"), the re-entering wave front resembles a spiral or top that continuously and rapidly rotates around a central core. ${ }^{20,21}$ Maintenance of spiral wave rotor activity depends on high cellular excitability and short atrial refractoriness. Drifting of rotor activity across the atria occurs with slow rotation speed, de-stabilizing electrical and positional stability and promoting its termination when reaching refractory tissue or anatomical boundaries.

Recent observations have suggested a more complex mechanism of AF, which is determined by patient-specific electrical and structural conditions (see below). Sources of ectopic activity located at the junction between the pulmonary veins and the left atrium have been revealed to initiate and perpetuate AF. Multiple-circuit left atrial re-entry then leads to fibrillatory conduction. The number of re-entrant circuits that can be accommodated depends on left atrial size and on excitation wavelength determined by refractory periods. Small atrial diameters and prolonged atrial refractoriness limits the quantity of functional circuits and prevents sustained AF.

\section{Electrical remodeling}

Evolving experimental and clinical evidence indicates that electrical and structural alterations referred to as atrial remodeling play an essential role in development and maintenance of AF. Remodeling may be caused either by concomitant cardiovascular disease or by the arrhythmia itself. The generation of electrical conditions and anatomical substrates that support slow conduction, shortening of atrial refractory periods, and electrical re-entry is mechanistically relevant. Electroanatomical substrates allow for generation of multiple re-entrant circuits, providing the basis for maintenance of persistent $\mathrm{AF}$ and stabilizing the arrhythmia. Action potential shortening and reduced refractoriness is associated with a reduction of L-type $\mathrm{Ca}^{2+}$ channel expression and up-regulation of inward rectifier $\left(I_{\mathrm{K} 1}\right)$ and acetylcholine-dependent $\mathrm{K}^{+}$current $\left(I_{\mathrm{K}, \mathrm{Ach}}\right)$. Spatiotemporal changes in $\mathrm{K}^{+}$ion channel expression that may occur with age or in response to cardiac disease including AF are particularly critical. ${ }^{22-24}$ Potassium channels determine atrial action potential duration and effective refractory periods, and both increased and reduced $\mathrm{K}^{+}$channel activity can lead to atrial arrhythmia. The significance of potassium channel dysfunction is well documented for ventricular arrhythmias. ${ }^{24,25}$ In addition, atrial fibrosis reduces conduction velocity locally, causes anisotropic conductivity, and represents an irreversible substrate for $\mathrm{AF}^{26}$ Additional structural changes observed during AF include inflammatory reaction, amyloid deposit, apoptosis, necrosis, hypertrophy, redistribution of gap junctions, microvascular changes, and endocardial remodeling. The molecular mechanisms leading to electroanatomical remodeling remain incompletely understood.

\section{Autonomic nervous system}

Cardiac electrophysiology is tightly regulated by the autonomic nervous system. The delicate balance between adrenergic and cholinergic stimulation influences the occurrence and maintenance of $\mathrm{AF}^{27}$ Adrenergic activity and increased catecholamine levels trigger AF acutely and are associated with chronic AF, while $\beta$-blockers are effective in suppressing the arrhythmia. Furthermore, abnormal heart rate variability indicating disturbances of cardiac autonomic regulation was identified as an independent risk factor of AF, and vagosympathetic nerve stimulation has proven effective to reduce AF and to prevent and reverse atrial remodeling induced by cholinergic stimulation. ${ }^{28,29}$ Electrophysiological effects of adrenergic and cholinergic stimuli are mediated via regulation of multiple cardiac ion currents, including pacemaker current, L-type $\mathrm{Ca}^{2+}$ current, and multiple potassium ion channels. ${ }^{27}$

\section{Treatment}

\section{Clinical classification}

Atrial fibrillation tends to progress from short episodes to longer more frequent attacks and sustained forms of arrhythmia. Asymptomatic episodes are common, even in symptomatic patients. Five types of AF are clinically distinguished based on the duration of the arrhythmia:

1. First diagnosed $A F$ is present in patients who experience AF for the first time.

2. Paroxysmal $A F$ terminates spontaneously within less than 7 days after onset.

3. Persistent $A F$ extends beyond 7 days or requires termination by cardioversion.

4. Long-standing persistent AF is diagnosed when persistent AF lasts $\geq 1$ year.

5. The term permanent $A F$ is used when the arrhythmia is accepted and no rhythm control strategy is followed.

Of note, the risk of thromboembolism does not depend on the duration of AF episodes. Thus, therapies aimed at preventing AF-related complications (eg, stroke) are similarly required in all cases of $\mathrm{AF}$.

\section{Therapeutic concepts}

AF therapy focuses on symptom management and prevention of complications and may require treatment of concomitant 
cardiac or endocrine disease, control of cardiac rhythm and ventricular rate, and antithrombotic therapy (Figure 2). The successful treatment of primary causes is essential to suppress or eliminate AF. During AF, normal AV node conduction can lead to rapid ventricular rate response, resulting in impairment of left ventricular function and severe limitation of physical activity, which is a frequent cause for patient hospitalization. Rate control is usually achieved by pharmacological reduction of AV nodal conduction velocity until the ventricular heart rate is decreased to a less symptomatic state. In contrast, rhythm control aims at converting the patient's heart rhythm to normal sinus rhythm (NSR) and at maintaining NSR once achieved. Prevention of thromboembolic complications is a major priority and reduces morbidity and mortality. For patients with increased bleeding risk, surgical exclusion or interventional occlusion of the left atrial appendage (LAA) are available.

\section{Current antiarrhythmic drug therapy}

Conditions that increase excitability and shorten refractoriness promote AF maintenance. Rhythm control drugs aiming at suppression of AF target these mechanisms. However, the number of compounds available for rhythm control is limited. Classic antiarrhythmic $\mathrm{Na}^{+}$current inhibitors (class I drugs) decrease excitability and destabilize rotator activity, while $\mathrm{K}^{+}$channel blocking (or class III) drugs suppress re-entry

\section{Treatment of atrial fibrillation}

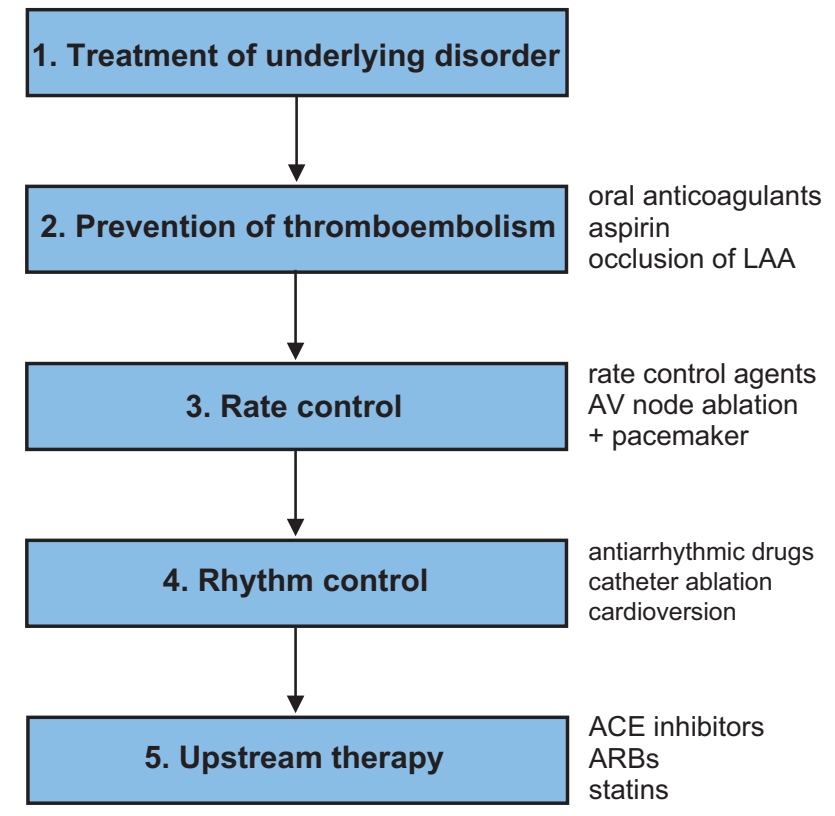

Figure 2 Overview: treatment options for patients with atrial fibrillation. Abbreviations: LAA, left atrial appendage; ACE, angiotensin-converting enzyme; $A R B$, angiotensin receptor blocker. mechanisms by extending action potential duration and repolarization. Class Ic agents increase mortality in patients with structural heart disease, and amiodarone harbors an extensive side effect profile despite its efficacy in maintaining sinus rhythm. Nonetheless amiodarone is the most frequently used antiarrhythmic drug to achieve and maintain normal sinus rhythm. In addition, amiodarone has a heart rate lowering effect and simultaneously can be used for rate control, particularly if classic rate control agents failed or are contraindicated. The use of amiodarone is limited by significant adverse effects including decreased blood pressure, pulmonary toxicity, skin discoloration, thyroid toxicity, corneal deposits, optic neuropathy, and sinus bradycardia. As a result, a significant number of patients are not eligible or refuse to take the drug. Furthermore, rhythm control by either of these compounds has not been shown to reduce patient mortality. Pharmacological rate control is usually achieved by suppression of calcium currents or by application of common drugs such as $\beta$-blockers or digitalis compounds. In a subset of AF patients, pharmacological interventions are not tolerated or inefficient. The remaining option in clinical practice is a non-specific and palliative last resort approach combining pacemaker implantation and AV node ablation. Despite representing a primary approach to pharmacological treatment of AF, current antiarrhythmic therapy displays low efficacy or harbors a poor safety profile, illustrating the need for novel drugs to treat AF. Consequently, pharmaceutical research has focused on the development of more favorable multichannel-blocking agents as well as novel ion-channel and non-channel targets. ${ }^{7,9}$

\section{Rate vs rhythm control}

Studies comparing rate and rhythm control strategies (PIAF [Pharmacological Intervention in Atrial Fibrillation]; AFFIRM [Atrial Fibrillation Follow-up Investigation of Rhythm Management]; RACE [Rate Control versus Electrical Cardioversion for Persistent Atrial Fibrillation]; STAF [The Strategies of Treatment of Atrial Fibrillation], and HOT CAFE [How to Treat Chronic Atrial Fibrillation]) did not prove morbidity/mortality benefits, and rhythm control using the class III drug amiodarone was associated with higher hospitalization rates. ${ }^{30-34}$ These findings are counterintuitive considering severe complications and mortality associated with AF but may be attributed to proarrhythmic side effects of antiarrhythmic drugs used for rhythm control. In clinical practice, the decision between rate or rhythm control depends on multiple patient-specific factors including the severity of symptoms, hemodynamic effects, duration and frequency of 
AF episodes, underlying structural or endocrine disease, and the outcome of previous treatment regimes.

\section{Acute pharmacological and direct current cardioversion}

Many episodes of AF terminate spontaneously within 24 to 48 hours. However, an inappropriate ventricular rate or irregular heartbeat associated with AF may require acute recovery of sinus rhythm in severely compromised patients. Acute conversion to NSR may be achieved either pharmacologically or by direct current cardioversion (DCC). In cases of hemodynamic instability, immediate DCC should be performed. In stable patients with recent onset AF (within less than 7 days) or in patients with persisting symptoms despite adequate rate control, pharmacological cardioversion may be useful. The acute success rate of pharmacological cardioversion is lower compared with direct current cardioversion (DCC), but sedation or anesthesia is not required. For pharmacological cardioversion, continuous medical supervision and ECG monitoring are required to detect rhythm disturbances such as ventricular arrhythmia, sinus node arrest, or atrioventricular block. Drugs available for pharmacological cardioversion include amiodarone, flecainide, and propafenone. Class Ic agents such as flecainide and propafenone may be administered intravenously or orally to terminate acute onset AF in stable patients. Cardioversion following amiodarone treatment typically takes at least several hours. Repeated oral pharmacological cardioversion ("pill in the pocket") using flecainide or propafenone is applicable in selected ambulatory patients, once safety and efficacy of this intervention have been established in a monitored setting.

The risk of thromboembolism associated with pharmacological or DC cardioversion requires anticoagulant therapy that depends on the onset of AF. ${ }^{1}$ Most patients with AF $<48$ hours in duration can be cardioverted on low molecular weight or unfractionated heparin without significant risk for stroke. If AF onset preceded cardioversion by $>48$ hours transesophageal echocardiography may be used to rule out intracardiac thrombi prior to acute cardioversion.

\section{Novel antiarrhythmic drugs}

Dronedarone is a new antiarrhythmic drug that has been developed to provide rhythm and rate control in AF patients, with fewer side effects compared with amiodarone. ${ }^{35}$ Dronedarone is chemically related to amiodarone but unlike amiodarone, it does not possess the iodine moieties affecting thyroid function. Moreover the addition of a methyl sulphonyl group decreases its lipophilicity and shortens its plasma half-life, thought to reduce organ toxicity due to cumulative effects. Similar to amiodarone, dronedarone is a multichannel blocker that meets criteria of all four Vaughan Williams anti-arrhythmic drug classes: rate-dependent inhibition of the rapid $\mathrm{Na}^{+}$current (class I), alpha and beta-adrenergic receptor inhibition (class II), blockade of $\mathrm{K}^{+}$outward currents as the main mechanism of action (class III), and blockade of slow $\mathrm{Ca}^{2+}$ inward currents (class IV). ${ }^{36-38}$ Action potential duration is prolonged, heart rate is reduced, and the risk of torsade de pointes arrhythmias is low. Dronedarone was approved in 2009 based on the results of the ATHENA trial (A PlaceboControlled, Double-Blind, Parallel Arm Trial to Assess the Efficacy of Dronedarone $400 \mathrm{mg}$ bid for the Prevention of Cardiovascular Hospitalization or Death from any Cause in Patients with Atrial Fibrillation/Atrial Flutter). ${ }^{39}$ Dronedarone significantly reduced the incidence of hospitalization due to cardiovascular events or death in high-risk patients with AF. Dronedarone represents a valuable addition to the limited spectrum of antiarrhythmic drugs and is currently recommended in patients with paroxysmal and persistent AF to achieve rate and rhythm control, excluding cases of severe or unstable congestive heart failure. Notably, liver function should be closely monitored as cases of severe liver damage have been reported in association with dronedarone. ${ }^{40}$

In 2010 the antiarrhythmic drug vernakalant was granted market approval for the conversion of recent-onset AF to sinus rhythm. ${ }^{41}$ The antiarrhythmic agent inhibits cardiac $\mathrm{Na}^{+}$ and $\mathrm{K}^{+}$channels including atrial-selective channels Kv1.5 $\left(I_{\mathrm{Kur}}\right)$ and Kir3.1/Kir3.4 $\left(I_{\mathrm{K}, \mathrm{Ach}}\right)$ and prolongs atrial refractoriness without significantly affecting ventricular effective refractory periods in humans. ${ }^{41-43}$ The AVRO (A Phase III Superiority Study of Vernakalant vs Amiodarone in Subjects with Recent Onset Atrial Fibrillation) trial indicated superiority of vernakalant compared with amiodarone for cardioversion within 90 minutes. ${ }^{44}$ Transient dysgeusia and sneezing are the most common adverse events in vernakalant patients, and significant proarrhythmia has not been reported.

Other drugs or drug targets are currently under investigation. In particular, potential atrial-selective targets that have previously received little attention are now studied intensely, including two-pore-domain potassium $\left(\mathrm{K}_{2 \mathrm{P}}\right)$ or transient receptor potential (TRP) channels, connexins, or proteins involved in calcium homeostasis. ${ }^{9,45-48}$

\section{Catheter ablation of atrial fibrillation}

Catheter ablation has become an established treatment modality in symptomatic AF patients. ${ }^{1}$ Interventional strategies of $\mathrm{AF}$ ablation isolate electrical triggers inside the pulmonary 
veins (ostial or circumferential pulmonary vein isolation), suppress excitation wave mobility and rotor activity by dividing atrial tissue into smaller, electrically isolated zones (creation of linear lesions), prevent re-entry and wavelet propagation by ablation of local areas of slow conduction ("complex fractionated atrial electrograms", CFAE), or eliminate autonomic ganglia by ablation. ${ }^{18,49-52}$ Circumferential pulmonary vein isolation using irrigated radiofrequency current is most commonly used to isolate ectopic electrical PV activity from the LA. More refined techniques for catheter ablation of AF are evolving rapidly to improve catheter manipulation, contact force monitoring, ablation efficacy, and procedural safety, while reducing procedure times, fluoroscopy exposure and physical demands. Ablation is currently recommended for patients with symptomatic AF despite antiarrhythmic treatment. Clinical presentation, left atrial size, previous therapy, or concomitant cardiovascular disease should be considered in each individual patient when evaluating catheter ablation. ${ }^{1}$ The recurrence rate in patients with paroxysmal AF is generally lower compared with persistent or permanent $A F .{ }^{53}$ Complications include pericardial tamponade, pericarditis, thromboembolic events, vascular complications, and pulmonary vein stenosis. ${ }^{2}$ In addition, esophageal injuries that depend on energy settings and esophageal temperature, as well as iatrogenic atrial septal defects caused by single-puncture, double transseptal approaches have been reported. Since the introduction of the technique the complication rate decreased while the success rate continuously increased owing to periprocedural safety measures and to improved operator skills and experience. ${ }^{54}$ Success rates of $\sim 80 \%$ have been reported in long-term studies that included multiple ablation procedures per patient. ${ }^{55}$ Mortality benefits of AF ablation have not yet been reported and are currently being investigated in a large multicenter trial (Catheter Ablation versus Antiarrhythmic Drug Therapy for Atrial Fibrillation, CABANA).${ }^{56}$

\section{Prevention of thromboembolic events in atrial fibrillation}

Atrial fibrillation increases the risk of stroke, and multiple studies have demonstrated that oral anticoagulation with vitamin $\mathrm{K}$ antagonists (VKA) is effective and more efficacious than aspirin for prevention of stroke in patients with AF. ${ }^{2,57-59}$ Recommendations for antithrombotic therapy are based on patient-specific evaluation of risk factors for stroke and systemic embolism. The simple $\mathrm{CHADS}_{2}$ (cardiac failure, hypertension, age, diabetes, and stroke [doubled]) risk stratification scheme may be used as initial and rapid means of assessing the risk of embolism. ${ }^{58,59}$ A more comprehensive approach is offered by the $\mathrm{CHA}_{2} \mathrm{DS}_{2}-\mathrm{VASc}$ score (congestive heart failure, hypertension, age $\geq 75$ years [doubled], diabetes, history of stroke or transient ischemic attack [doubled], vascular disease, age 65-74, and sex category [female]) which considers additional risk factors that may be required in cases of low to moderate risk as assessed by $\mathrm{CHADS}_{2} \cdot{ }^{60}$ Chronic oral anticoagulation therapy is recommended in patients with a $\mathrm{CHADS}_{2}$ or $\mathrm{CHA}_{2} \mathrm{DS}_{2}$-VASc score $\geq 2$ to achieve an international normalized ratio (INR) value in the range of 2-3 unless contraindicated. ${ }^{1}$ In patient with a $\mathrm{CHADS}_{2}$ score of $0-1$ additional risk stratification using the $\mathrm{CHA}_{2} \mathrm{DS}_{2}$-VASc scheme is recommended. Patients who exhibit no $\mathrm{CHA}_{2} \mathrm{DS}_{2}-\mathrm{VASc}$ risk factors may receive either no antithrombotic therapy or aspirin 75-325 mg daily. If one clinically relevant risk factor is present $\left(\mathrm{CHA}_{2} \mathrm{DS}_{2}-\mathrm{VASc}=1\right)$ oral anticoagulation $(\mathrm{OAC})$ therapy should be preferred over aspirin 75-325 mg daily. In all cases where OAC treatment is indicated, an assessment of bleeding complications is necessary to secure safety of OAC administration. Available risk scores that have been validated in anticoagulated patients include HAS-BLED (hypertension, abnormal renal/liver function, stroke, bleeding history, labile INR, elderly [>65 years], drugs/alcohol) and HEMORR 2 HAGES (hepatic or renal disease, ethanol abuse, malignancy, older [ $>75$ years], reduced platelet count or function, rebleeding risk [doubled], hypertension [uncontrolled], anemia, genetic factors [CYP 2C9 single nucleotide polymorphisms], excessive fall risk, stroke). ${ }^{61,62}$ The addition of clopidogrel to aspirin may be considered in patients with AF in whom OAC therapy is indicated but considered unsuitable. ${ }^{3}$

\section{New anticoagulant agents}

Vitamin $\mathrm{K}$ antagonists are cumbersome to use, require laboratory monitoring, and exhibit multiple interactions with food and drugs, resulting in significant discontinuation rates. The search for new oral anticoagulant drugs that are effective, safe and convenient to use has focused primarily on direct thrombin inhibitors (eg, dabigatran) and factor Xa inhibitors (eg, rivaroxaban, apixaban). The RE-LY (Randomized Evaluation of Long-Term Anticoagulant Therapy) study demonstrated non-inferiority of $110 \mathrm{mg}$ twice a day dabigatran to VKA for prevention of stroke and systemic embolism with lower rates of major hemorrhage. ${ }^{63}$ Furthermore, an increased dose (150 mg twice a day) exhibited lower rates of embolism and similar incidence of hemorrhage. Following its approval for prevention of 
thromboembolism in AF, dabigatran may be considered as an alternative to VKA therapy. Recommended dosage is $150 \mathrm{mg}$ twice a day in the US and depends on individual bleeding risk factors in Europe where approval is pending at the time of writing. ${ }^{1}$ The factor Xa inhibitor apixaban was superior to aspirin in AF patients intolerant or unsuitable for VKA in the AVERROES (Apixaban versus Acetylsalicylic Acid (ASA) to Prevent Strokes) trial, leading to premature termination of the study. ${ }^{64}$ Publication of these results is pending, and the non-inferiority study ARISTOTLE (Apixaban for Reduction in Stroke and other Thromboembolic Events in Atrial Fibrillation) is currently being conducted to compare apixaban to VKA.$^{65}$ In late 2010, results of the Stroke Prevention using the Oral Direct Factor Xa Inhibitor Rivaroxaban Compared with Warfarin in Patients with Nonvalvular Atrial Fibrillation (ROCKET AF) trial were presented at the American Heart Association's Scientific Sessions. ${ }^{66}$ The trial showed that the factor Xa inhibitor rivaroxaban was as effective as warfarin in preventing stroke in AF patients and did not increase their risk of bleeding. In the per protocol analysis, the rate of stroke and systemic embolism was lower in the rivaroxaban group than in the warfarin group, whereas in the intention-to-treat analysis the superiority of rivaroxaban over warfarin did not achieve statistical significance. These studies demonstrate that effective and safe alternatives to VKA may improve and facilitate anticoagulant therapy in the future.

\section{Interventional approaches to stroke prevention}

Approximately 90\% of thromboembolisms associated with AF are caused by thrombi originating from the left atrial appendage. Occlusion of the LAA should provide a non-pharmacological alternative to minimizing the risk of stroke in AF patients. However, LAA exclusion during surgery is not sufficiently supported by data and may even increase the risk of stroke if incomplete occlusion occurs, shifting the emphasis towards interventional strategies. ${ }^{67,68}$ Recently, studies of percutaneous transcatheter delivery of dedicated occlusion devices (PLAATO system [withdrawn due to commercial reasons], WATCHMAN, AMPLATZER cardiac plug) have produced encouraging results as alternatives to VKA in selected patients. ${ }^{69-72}$ In particular, LAA occlusion provides a therapeutic option in high-risk patients with contraindications to chronic anticoagulation. Limitations arise from procedural complications such as pericardial effusion, major bleeding, or device embolization.

\section{Upstream therapy}

Upstream therapy to reverse or inhibit myocardial remodeling associated with hypertension and heart failure may prevent the development of AF (primary prevention) or reduce recurrence rates and progression to permanent $\mathrm{AF}$ (secondary prevention). Upstream therapy is expected to reduce left atrial size, thereby limiting the number of functional reentrant circuits. In addition, upstream therapy may prevent re-entry by deceleration of intercellular conduction velocity and by suppression of electrical ion channel remodeling. Angiotensin-converting enzyme inhibitors, angiotensin II receptor blockers, and statins may be considered for primary prevention in patients with concomitant structural heart disease or for secondary prevention in cases of recurrent AF despite antiarrhythmic drug therapy. ${ }^{1,2}$ Evidence for upstream therapy for prevention of trial remodeling still remains controversial.

\section{Biological antiarrhythmic therapy}

Current pharmacotherapy to treat AF is limited by reduced efficacy, side effects, and safety issues in a significant number of patients. Non-pharmacological therapy is improving, but only a restricted number of patients can be treated by AF ablation. In search of novel treatment modalities, gene therapy offers greater selectivity than small-molecule or interventional approaches. ${ }^{10}$ The gene of interest is commonly packaged into adenovirus and delivered to the target area via direct injection or catheterbased interventional techniques, providing the advantage of site-restricted action in contrast to systemic application of drugs. ${ }^{10,73}$ Antiarrhythmic gene therapy targeting rate control has been evaluated in a porcine model of AF. Knock-down of an activating component of the adrenergic signalling pathway by over-expression of inhibitory $\mathrm{G}$ protein $\left(\mathrm{G \alpha}_{\mathrm{i} 2}\right)$, administered via percutaneous access and cardiac catheterization, reduced mean ventricular heart rates by $15 \%$ to $25 \% .{ }^{74,75}$ Gene transfer of the guanine nucleotide-binding proteins (GTP) binding protein Gem allows for control of heart rates during AF, independent of adrenergic signal transduction. Here AV nodal conduction is decreased by calcium current suppression via genetic inhibition of calcium channel trafficking to the plasma membrane. ${ }^{73}$ Thus, targeted biological modification of atrioventricular conduction may represent a viable strategy for heart rate control in AF. Biological rhythm control was achieved in a pre-clinical study by direct atrial gene transfer of a dominant-negative $\mathrm{K}^{+}$channel mutant, hERG G628S. ${ }^{76}$ Inactivation of hERG channels results in reduced repolarizing $I_{\mathrm{Kr}}$ current and action potential prolongation and successfully suppressed pacing-induced AF in a porcine model. 


\section{Conclusion}

Effective treatment of AF still remains an unmet medical need associated with high epidemiological and clinical relevance. Recent clinical and basic research efforts have revealed insights into fundamental mechanisms contributing to the arrhythmia. Heart diseases may induce progressive structural and electrical remodeling of the atria that promote AF by creating both substrate and electrophysiological properties for re-entry. After the onset of AF, atrial alterations are observed within 24 to 48 hours as a consequence of AF. These changes are associated with reduced electrical conduction and shortening of effective refractory periods, resulting in perpetuation of the arrhythmia. Multifactorial etiology and pathogenesis of AF require multimodal treatment of the disease, tailored to comprehensively target patient-specific mechanisms. The choice between frequency and rhythm control regimes depends on individual symptoms and prerequisites of each patient. Current antiarrhythmic drugs display either reduced efficiency or limited safety. Dronedarone represents a valuable addition to the limited spectrum of antiarrhythmic drugs and is currently recommended in patients with paroxysmal and persistent AF to achieve rate and rhythm control, excluding cases of severe or unstable congestive heart failure. Catheter ablation has become an established treatment modality in symptomatic AF patients. Efforts to further refine interventional techniques and to improve efficacy and safety of AF ablation are under way. Antithrombotic therapy is critical and should be based on individual risk factors for stroke/thrombembolism (eg, $\mathrm{CHA}_{2} \mathrm{DS}_{2}$-VASc score) and bleeding (eg, HAS-BLED). Oral compounds such as dabigatran or rivaroxaban have been developed to replace vitamin $\mathrm{K}$ antagonists and may become more widely available in the near future. Pharmacological upstream therapy using statins, angiotensin-converting enzyme inhibitors, or angiotensin receptor blockers could serve as valuable adjuvant treatment option in select cases. Finally, novel ion channel targets and antiarrhythmic gene therapy preventing substrate development and electrophysiological remodeling in AF are currently established and evaluated in pre-clinical studies to optimize treatment of the most debilitating of arrhythmias.

\section{Disclosure}

This work was supported in part by grants from the MaxPlanck-Society (TANDEM project to PAS) and from the German Heart Foundation/German Foundation for Heart Research (to DT). PAS reports receiving travel grants from St Jude Medical. DT serves on advisory boards for sanofi-aventis and reports receiving travel grants or lecture fees from Boehringer Ingelheim Pharma, Boston Scientific, Hansen Medical, Medtronic, Sorin Group, and St Jude Medical. The remaining authors declare that they have no conflict of interest.

\section{References}

1. European Heart Rhythm Association, European Association for CardioThoracic Surgery; Camm AJ, et al. Guidelines for the management of atrial fibrillation: the Task Force for the Management of Atrial Fibrillation of the European Society of Cardiology (ESC). Eur Heart J. 2010;31(19):2369-2429.

2. Fuster V, Rydén LE, Cannom DS, et al. ACC/AHA/ESC 2006 Guidelines for the Management of Patients with Atrial Fibrillation: a report of the American College of Cardiology/American Heart Association Task Force on Practice Guidelines and the European Society of Cardiology Committee for Practice Guidelines (Writing Committee to Revise the 2001 Guidelines for the Management of Patients With Atrial Fibrillation): developed in collaboration with the European Heart Rhythm Association and the Heart Rhythm Society. Circulation. 2006;114(7):e257-e354.

3. Wann LS, Curtis AB, January CT, et al. 2011 ACCF/AHA/HRS focused update on the management of patients with atrial fibrillation (updating the 2006 guideline): a report of the American College of Cardiology Foundation/American Heart Association Task Force on Practice Guidelines. Circulation. 2011;123(1):104-123.

4. Psaty BM, Manolio TA, Kuller LH, et al. Incidence of and risk factors for atrial fibrillation in older adults. Circulation. 1997;96(7):2455-2461.

5. Benjamin EJ, Wolf PA, D’Agostino RB, Silbershatz H, Kannel WB, Levy D. Impact of atrial fibrillation on the risk of death: the Framingham Heart Study. Circulation. 1998;98(10):946-952.

6. Komatsu T, Tachibana H, Sato Y, Ozawa M, Kunugida F, Nakamura M. Efficacy of antiarrhythmic drug therapy in preventing recurrence of atrial fibrillation and long-term cardiovascular prognosis in patients with asymptomatic paroxysmal atrial fibrillation. Int Heart $J$. 2010;51(2):98-104.

7. Dobrev D, Nattel S. New antiarrhythmic drugs for treatment of atrial fibrillation. Lancet. 2010;375(9721):1212-1223.

8. Nattel S. New ideas about atrial fibrillation 50 years on. Nature. 2002;415(6868):219-226.

9. Ravens U. Novel pharmacological approaches for antiarrhythmic therapy. Naunyn Schmiedebergs Arch Pharmacol. 2010;381(3): 187-193.

10. Cho HC, Marbán E. Biological therapies for cardiac arrhythmias: can genes and cells replace drugs and devices? Circ Res. 2010;106(4): 674-685.

11. Hodgson-Zingman DM, Karst ML, Zingman LV, et al. Atrial natriuretic peptide frameshift mutation in familial atrial fibrillation. N Engl J Med. 2008;359(2):158-165.

12. Olson TM, Michels VV, Ballew JD, et al. Sodium channel mutations and susceptibility to heart failure and atrial fibrillation. JAMA. 2005;293(4):447-454.

13. Chen YH, Xu SJ, Bendahhou S, et al. KCNQ1 gain-of-function mutation in familial atrial fibrillation. Science. 2003;299(5604):251-254.

14. Yang Y, Xia M, Jin Q, et al. Identification of a KCNE2 gain-of-function mutation in patients with familial atrial fibrillation. Am J Hum Genet. 2004;75(5):899-905.

15. Gudbjartsson DF, Holm H, Gretarsdottir S, et al. A sequence variant in ZFHX3 on 16q22 associates with atrial fibrillation and ischemic stroke. Nat Genet. 2009;41(8):876-878.

16. Benjamin EJ, Rice KM, Arking DE, et al. Variants in ZFHX3 are associated with atrial fibrillation in individuals of European ancestry. Nat Genet. 2009;41(8):879-881.

17. Ellinor PT, Lunetta KL, Glazer NL, et al. Common variants in $\mathrm{KCNN} 3$ are associated with lone atrial fibrillation. Nat Genet. 2010;42(3):240-244. 
18. Haissaguerre M, Jais P, Shah DC, et al. Spontaneous initiation of atrial fibrillation by ectopic beats originating in the pulmonary veins. $N E n g l$ J Med. 1998;339(10):659-666.

19. Allessie MA, Bonke FI, Schopman FJ. Circus movement in rabbit atrial muscle as a mechanism of tachycardia III. The "leading circle" concept: a new model of circus movement in cardiac tissue without the involvement of an anatomical obstacle. Circ Res. 1977;41(1):9-18.

20. Davidenko JM, Pertsov AV, Salomonsz R, Baxter W, Jalife J. Stationary and drifting spiral waves of excitation in isolated cardiac muscle Nature. 1992;355(6358):349-351.

21. Pertsov AM, Davidenko JM, Salomonsz R, Baxter WT, Jalife J. Spiral waves of excitation underlie reentrant activity in isolated cardiac muscle. Circ Res. 1993;72(3):631-650.

22. Varro A, Biliczki P, Lost N, Virag L, Hala O, Papp JG. Pharmacology of potassium channel blockers. In: Papp JG, Straub M, Ziegler D, editors. Atrial Fibrillation: New Therapeutic Concepts. Amsterdam: IOS Press/ Ohmsa; 2003:27-39.

23. Nattel S, Maguy A, Le Bouter S, Yeh YH. Arrhythmogenic ion-channel remodeling in the heart: heart failure, myocardial infarction, and atrial fibrillation. Physiol Rev. 2007;87(2):425-456.

24. Ravens U, Cerbai E. Role of potassium currents in cardiac arrhythmias. Europace. 2008;10(10):1133-1137.

25. Thomas D, Karle CA, Kiehn J. The cardiac hERG/IKr potassium channel as pharmacological target: structure, function, regulation, and clinical applications. Curr Pharm Des. 2006;12(18):2271-2283.

26. Allessie M, Ausma J, Schotten U. Electrical, contractile and structural remodeling during atrial fibrillation. Cardiovasc Res. 2002;54(2):230-246.

27. Workman AJ. Cardiac adrenergic control and atrial fibrillation. Naunyn Schmiedebergs Arch Pharmacol. 2010;381(3):235-249.

28. Jons C, Raatikainen P, Gang UJ, et al. Autonomic dysfunction and new-onset atrial fibrillation in patients with left ventricular systolic dysfunction after acute myocardial infarction: a CARISMA substudy J Cardiovasc Electrophysiol. 2010;21(9):983-990.

29. Sheng X, Scherlag BJ, Yu L, et al. Prevention and reversal of atrial fibrillation inducibility and autonomic remodeling by low-level vagosympathetic nerve stimulation. J Am Coll Cardiol. 2011;57(5): 563-571.

30. Carlsson J, Miketic S, Windeler J, et al. Randomized trial of rate-control versus rhythm-control in persistent atrial fibrillation: the Strategies of Treatment of Atrial Fibrillation (STAF) study. J Am Coll Cardiol. 2003;41(5):1690-1696.

31. Hohnloser SH, Kuck KH, Lilienthal J. Rhythm or rate control in atrial fibrillation - Pharmacological Intervention in Atrial Fibrillation (PIAF) a randomized trial. Lancet. 2000;356(9244):1789-1794.

32. Van Gelder IC, Hagens VE, Bosker HA, et al. A comparison of rate control and rhythm control in patients with recurrent persistent atrial fibrillation. $N$ Engl J Med. 2002;347(23):1834-1840.

33. Wyse DG, Waldo AL, DiMarco JP, et al. Atrial Fibrillation Follow-up Investigation of Rhythm Management (AFFIRM) Investigators. A comparison of rate control and rhythm control in patients with atrial fibrillation. N Engl J Med. 2002;347(12):1825-1833.

34. Opolski G, et al. Rate control vs rhythm control in patients with nonvalvular persistent atrial fibrillation: the results of the Polish How to Treat Chronic Atrial Fibrillation (HOT CAFE) Study. Chest. 2004;126(2):476-486.

35. Wolbrette D, Gonzalez M, Samii S, Banchs J, Penny-Peterson E, Naccarelli G. Dronedarone for the treatment of atrial fibrillation and atrial flutter: approval and efficacy. Vasc Health Risk Manag. 2010;6: 517-523.

36. Thomas D, Kathofer S, Zhang W, et al. Acute effects of dronedarone on both components of the cardiac delayed rectifier $\mathrm{K}^{+}$current, HERG and KvLQT1/minK potassium channels. Br J Pharmacol. 2003;140(5):996-1002.

37. Kathofer S, Thomas D, Karle CA. The novel antiarrhythmic drug dronedarone: comparison with amiodarone. Cardiovasc Drug Rev. 2005;23(3):217-230.
38. Schweizer PA, Becker R, Katus HA, Thomas D. Dronedarone: current evidence for its safety and efficacy in the management of atrial fibrillation. Drug Des Devel Ther. 2011;5:27-39.

39. Hohnloser SH, Crijns HJ, van Eickels M, et al. Effect of dronedarone on cardiovascular events in atrial fibrillation. $N$ Engl J Med. 2009;360(7):668-678.

40. FDA.gov [homepage on the internet]. Rockville: US Food and Drug Administration; FDA Drug Safety Communication: Severe liver injury associated with the use of dronedarone (marketed as Multaq) [updated January 20, 2011]. http://www.fda.gov/Drugs/DrugSafety/ucm240011. htm. Accessed February 5, 2011.

41. Dobrev D, Hamad B, Kirkpatrick P. Vernakalant. Nat Rev Drug Discov. 2010;9(12):915-916.

42. Fedida D, Orth PM, Chen JY, et al. The mechanism of atrial antiarrhythmic action of RSD1235. J Cardiovasc Electrophysiol. 2005;16(11): $1227-1238$.

43. Dorian P, Pinter A, Mangat I, Korley V, Cvitkovic SS, Beatch GN. The effect of vernakalant (RSD1235), an investigational antiarrhythmic agent, on atrial electrophysiology in humans. J Cardiovasc Pharmacol. 2007;50(1):35-40.

44. Camm AJ, Capucci A, Hohnloser SH, et al. A randomized activecontrolled study comparing the efficacy and safety of vernakalant to amiodarone in recent-onset atrial fibrillation. J Am Coll Cardiol. 2011;57(3):313-321.

45. Gierten J, Ficker E, Bloehs R, et al. Regulation of two-pore-domain $\left(\mathrm{K}_{2 \mathrm{P}}\right)$ potassium leak channels by the tyrosine kinase inhibitor genistein. Br J Pharmacol. 2008;154(8):1680-1690.

46. Gierten J, Ficker E, Bloehs R, et al. The human cardiac $\mathrm{K}_{2 \mathrm{P}} 3.1$ (TASK-1) potassium leak channel is a molecular target for the class III antiarrhythmic drug amiodarone. Naunyn Schmiedebergs Arch Pharmacol. 2010;381(3):261-270.

47. Dhein S, Hagen A, Jozwiak J, et al. Improving cardiac gap junction communication as a new antiarrhythmic mechanism: the action of antiarrhythmic peptides. Naunyn Schmiedebergs Arch Pharmacol. 2010;381(3):221-234.

48. Dobrev D. Atrial $\mathrm{Ca}^{2+}$ signaling in atrial fibrillation as an antiarrhythmic drug target. Naunyn Schmiedebergs Arch Pharmacol. 2010;381(3):195-206.

49. Jaïs P, Shah DC, Haïssaguerre M, et al. Efficacy and safety of septal and left-atrial linear ablation for atrial fibrillation. Am J Cardiol. 1999;84(9A):139R-146R.

50. Pappone $\mathrm{C}$, Rosanio $\mathrm{S}$, Oreto $\mathrm{G}$, et al. Circumferential radiofrequency ablation of pulmonary vein ostia: A new anatomic approach for curing atrial fibrillation. Circulation. 2000;102(21): 2619-2628.

51. Nademanee K, McKenzie J, Kosar E, et al. A new approach for catheter ablation of atrial fibrillation: mapping of the electrophysiologic substrate. J Am Coll Cardiol. 2004;43(11):2044-2053.

52. Pokushalov E, Romanov A, Artyomenko S, et al. Ganglionated plexi ablation for longstanding persistent atrial fibrillation. Europace. 2010;12(3):342-346.

53. Gaita F, Caponi D, Scaglione M, et al. Long-term clinical results of 2 different ablation strategies in patients with paroxysmal and persistent atrial fibrillation. Circ Arrhythm Electrophysiol. 2008;1(4): 269-275.

54. Takahashi A. Catheter ablation is established as a treatment option for atrial fibrillation - is catheter ablation established as a treatment option of atrial fibrillation? (Pro). Circ J. 2010;74(9): 1972-1977.

55. Ouyang F, Tilz R, Chun J, et al. Long-term results of catheter ablation in paroxysmal atrial fibrillation: lessons from a 5-year follow-up. Circulation. 2010;122(23):2368-2377.

56. Jaïs P, Packer DL. Ablation vs drug use for atrial fibrillation. Eur Heart J Suppl. 2007;9(Suppl G):G26-G34.

57. Hart RG, Benavente O, McBride R, Pearce LA. Antithrombotic therapy to prevent stroke in patients with atrial fibrillation: a meta-analysis. Ann Intern Med. 1999;131(7):492-501. 
58. Gage BF, Waterman AD, Shannon W, Boechler M, Rich MW, Radford MJ. Validation of clinical classification schemes for predicting stroke: results from the National Registry of Atrial Fibrillation. JAMA. 2001;285(22):2864-2870.

59. Gage BF, van Walraven C, Pearce L, et al. Selecting patients with atrial fibrillation for anticoagulation: stroke risk stratification in patients taking aspirin. Circulation. 2004;110(16):2287-2292.

60. Lip GY, Nieuwlaat R, Pisters R, Lane DA, Crijns HJ. Refining clinical risk stratification for predicting stroke and thromboembolism in atrial fibrillation using a novel risk factor-based approach: the euro heart survey on atrial fibrillation. Chest. 2010;137(2):263-272.

61. Pisters R, Lane DA, Nieuwlaat R, de Vos CB, Crijns HJ, Lip GY. A novel user-friendly score (HAS-BLED) to assess 1-year risk of major bleeding in patients with atrial fibrillation: the Euro Heart Survey. Chest. 2010;138(5):1093-1100.

62. Gage BF, Yan Y, Milligan PE, Waterman AD, Culverhouse R, Rich MW, et al. Clinical classification schemes for predicting hemorrhage: results from the National Registry of Atrial Fibrillation (NRAF). Am Heart J. 2006;151(3):713-719.

63. Connolly SJ, Ezekowitz MD, Yusuf S, et al. Dabigatran versus warfarin in patients with atrial fibrillation. $N$ Engl J Med. 2009;361(12): 1139-1151.

64. Eikelboom JW, O’Donnell M, Yusuf S, et al. Rationale and design of AVERROES: apixaban versus acetylsalicylic acid to prevent stroke in atrial fibrillation patients who have failed or are unsuitable for vitamin K antagonist treatment. Am Heart J. 2010;159(3): 348-353.e1

65. Lopes RD, Alexander JH, Al-Khatib SM, et al. Apixaban for reduction in stroke and other Thromboembolic events in atrial fibrillation (ARISTOTLE) trial: design and rationale. Am Heart J. 2010;159(3): 331-339.

66. ROCKET AF Study Investigators. Rivaroxaban-once daily, oral, direct factor Xa inhibition compared with vitamin $\mathrm{K}$ antagonism for prevention of stroke and Embolism Trial in Atrial Fibrillation: rationale and design of the ROCKET AF study. Am Heart J. 2010;159(3):340-347.e1.
67. Dawson AG, Asopa S, Dunning J. Should patients undergoing cardiac surgery with AF have left atrial appendage exclusion? Interact Cardiovasc Thorac Surg. 2010;10(2):306-311.

68. Kanderian AS, Gillinov AM, Pettersson GB, Blackstone E, Klein AL. Success of surgical left atrial appendage closure: assessment by transesophageal echocardiography. J Am Coll Cardiol. 2008; 52(11):924-929.

69. Ostermayer SH, Reisman M, Kramer PH, et al. Percutaneous left atrial appendage transcatheter occlusion (PLAATO system) to prevent stroke in high-risk patients with non-rheumatic atrial fibrillation: results from the international multi-center feasibility trials. J Am Coll Cardiol. 2005;46(1):9-14.

70. Sick PB, Schuler G, Hauptmann KE, et al. Initial worldwide experience with the WATCHMAN left atrial appendage system for stroke prevention in atrial fibrillation. $J$ Am Coll Cardiol. 2007;49(13):1490-1495.

71. Holmes DR, Reddy VY, Turi ZG, et al. Percutaneous closure of the left atrial appendage versus warfarin therapy for prevention of stroke in patients with atrial fibrillation: a randomised non-inferiority trial. Lancet. 2009;374(9689):534-542.

72. Block PC, Burstein S, Casale PN, et al. Percutaneous left atrial appendage occlusion for patients in atrial fibrillation suboptimal for warfarin therapy: 5-year results of the PLAATO (Percutaneous Left Atrial Appendage Transcatheter Occlusion) Study. JACC Cardiovasc Interv. 2009;2(7):594-600.

73. Marbán E. Cardiac channelopathies. Nature. 2002;415(6868): 213-218.

74. Donahue JK, Heldman AW, Fraser H, et al. Focal modification of electrical conduction in the heart by viral gene transfer. Nat Med. 2000;6(12):1395-1398.

75. Bauer A, McDonald AD, Nasir K, et al. Inhibitory G protein overexpression provides physiologically relevant heart rate control in persistent atrial fibrillation. Circulation. 2004;110(19):3115-3120.

76. Amit G, Kikuchi K, Greener ID, Yang L, Novack V, Donahue JK. Selective molecular potassium channel blockade prevents atrial fibrillation. Circulation. 2010;121(21):2263-2270.
Vascular Health and Risk Management

\section{Publish your work in this journal}

Vascular Health and Risk Management is an international, peerreviewed journal of therapeutics and risk management, focusing on concise rapid reporting of clinical studies on the processes involved in the maintenance of vascular health; the monitoring, prevention and treatment of vascular disease and its sequelae; and the involvement of

\section{Dovepress}

metabolic disorders, particularly diabetes. This journal is indexed on PubMed Central and MedLine. The manuscript management system is completely online and includes a very quick and fair peer-review system, which is all easy to use. Visit http://www.dovepress.com/ testimonials.php to read real quotes from published authors. 